\title{
Promotion de la recherche en médecine de premier recours: un investissement pour le futur
}

La production de recherche en médecine de premier recours est médiocre en Suisse comparé avec certains pays d'Europe tels que la Hollande, l'Angleterre, le Danemark et autres. Souligné lors d'un séminaire organisé par l'Académie Suisse des Sciences Médicale (ASSM) en novembre 2002, cette recherche est cependant de toute importance étant donné que la plus grande partie des rencontres médecin-malade ont lieu dans le cabinet et que la situation contextuelle et culturelle du praticien est bien différente de celle de l'hôpital, où s'effectue la majeure partie de la recherche clinique et d'où viennent, par la suite, les recommandations pour la pratique.

Cependant, ni le praticien ni l'institution académique ne peuvent seuls améliorer la qualité de cette recherche. Les observations pertinentes surgissent chez le praticien et doivent y être formulées en questions de recherche. Le praticien n'étant souvent pas familier avec les méthodologies de recherche, il ne sait pas dresser un plan de recherche et n'a pas l'habitude des multiples méandres menant à une publication. L'institution doit donc lui venir en aide. Cependant, par manque de contact avec les problèmes du praticien, les conseils et soutiens de celle-ci sont souvent inadaptés. La solution semble donc claire: par une formation appropriée, il faut d'une part augmenter le nombre de praticiens pour les rendre familiers (mais pas nécessairement experts) en méthodes de recherche leur permettant d'avoir une discussion d'égal à égal avec l'institution et, d'autre part, s'assurer que les chercheurs dans les institutions de recherche (policliniques de médecine, instituts d'épidé- miologie clinique et autres) aient une expérience de la pratique quotidienne. Cette solution passe par la formation continue, telle qu'elle est proposée aujourd'hui dans de nombreux domaines.

L'ASSM, qui depuis près de vingt ans soutient la recherche en médecine appliquée, est également prête à venir en aide pour la formation de futurs praticiens chercheurs dont l'activité se déroulera majoritairement au cabinet. Comment procéder? Idéalement, un nouveau curriculum de recherche de médecine de premier recours serait créé en Suisse sous forme de certificat ou de maîtrise. De telles solutions ont été trouvées dans d'autres domaines en Suisse et ont été réalisées dans d'autres pays, mais elles sont coûteuses et difficiles à mettre en pratique. Remarquons que certains modules de formation en recherche clinique existent déjà chez nous, surtout en Suisse romande. Il faudrait en créer ailleurs et les adapter aux besoins de la médecine de premier recours. Les unités de médecine générale (FIAM) pourraient y jouer un rôle important. Il faut aussi avoir un lieu de coordination pour renseigner les praticiens qui veulent se former en recherche, discuter avec les enseignants, proposer des modules à la pratique de premier recours, canaliser les soutiens financiers aux candidats, bref, un lieu où sponsors, enseignants et praticiens puissent se réunir. Le Collège de Médecine de Premier Recours, où les différents acteurs sont représentés, nous semble l'endroit prédestiné pour entrer en action.

Prof. Hans Stalder, Département de Médecine Communautaire, Hôpital Cantonal, Genève 


\section{Prolongation de la clause du besoin en lieu et place de levée de l'obligation de contracter: tomber de Charybde en Scylla?}

La Commission de la sécurité sociale et de la santé publique du Conseil des Etats préconise de prolonger le blocage de l'accès à la pratique privée, tel qu'il a été introduit il y a tout juste trois ans, en été 2002, sur la base de l'art. 55a de la LAMal.

A cette nouvelle, certains esprits malins ont manifestement espéré que le corps médical se sentirait soulagé: Ouf! Nous avons eu de la chance ... Ce n'est pas la levée de l'obligation de contracter que le législateur veut imposer, mais la prolongation - pour une durée de trois ans du gel des admissions qui, selon ses concepteurs, $\mathrm{a}$ «fait ses preuves». Et d'ici là, l'abolition du libre choix du médecin sentira de toute façon le réchauffé, car la pénurie de praticiens sera devenue si évidente que les politiques devront plutôt s'interroger sur la voie à suivre pour rendre la profession médicale plus attrayante.

Ne soyons pas dupes. La prolongation du gel des admissions ne saurait en aucun cas servir d'«alternative» à la levée de l'obligation de contracter débattue par le Parlement: il est prouvé que ce blocage n'a rien apporté du point de vue des coûts et nous savons qu'il n'apportera rien de plus s'il est prolongé. En revanche, il mine irrémédiablement l'attrait de la profession et contribue à aggraver la pénurie de médecins qui s'annonce de toute manière. Le DFI luimême a publié une étude qui démontre l'absence d'effets positifs de la clause du besoin... et nous en connaissons bien les «effets secondaires»!
Ni le blocage de l'accès à la pratique privée ni la suppression du libre choix du médecin ne sont aptes à influencer positivement l'évolution des coûts de la santé. En effet, ce n'est pas le nombre de prestataires de soins qu'il s'agirait de contrôler, mais bien l'efficacité et l'efficience des prestations fournies. Toute autre solution n'a aucun sens et risque d'affecter négativement, de manière directe et incontrôlée, la qualité des soins de santé.

L'ASMAC Suisse se prépare à lancer un référendum contre la prolongation du gel des admissions. Elle a en outre d'ores et déjà décidé de soutenir un éventuel référendum de la FMH contre la levée de l'obligation de contracter.

Nous ne sommes pourtant pas des «Neinsager».

Mais un oui ou, pire encore, un silence de notre part en ce qui concerne la prolongation de la clause du besoin, reviendrait à un «oui» à l'adresse des Chambres fédérales, quant à la levée de l'obligation de contracter.

L'ASMAC et la FMH adopteront une position commune sur ces questions. Une scission du corps médical ne saurait qu'être un rêve de quelques politiciens qui prennent leurs désirs pour des réalités. La seule réalité, c’est la cohésion du corps médical. 


\section{Verlängerung des Zulassungsstopps statt Aufhebung des Kontrahierungszwangs: vom Regen in die Traufe?}

Die ständerätliche Kommission für Soziale Sicherheit und Gesundheit schlägt die Verlängerung des Zulassungsstopps, wie er vor knapp drei Jahren im Sommer 2002 auf der Grundlage von Art. 55a KVG eingeführt wurde, vor.

Gewisse Kreise haben offenbar gehofft, dass damit ein Aufatmen durch die Reihen der Ärzteschaft gehen würde: Glück gehabt - nicht die Aufhebung des Kontrahierungszwangs wird vom Gesetzgeber vorangetrieben, sondern die Verlängerung des «bewährten» Zulassungsstopps für junge Ärztinnen und Ärzte, und zwar für weitere drei Jahre. Und bis dann wäre die Aufhebung der freien Arztwahl ohnehin kalter Kaffee, da der Ärztemangel derart deutlich sichtbar sein wird, dass die Politik sich mit der Frage wird befassen müssen, wie sie die Attraktivität des Arztberufes steigern kann.

Machen wir uns aber nichts vor: Die Verlängerung des Zulassungsstopps - einer Massnahme, die erwiesenermassen auf der Kostenseite nichts gebracht hat und auch im Falle einer Verlängerung nichts bringen wird, dafür an der Attraktivität des Arztberufes unaufhaltsam nagt und zu einer Beschleunigung des ohnehin sich abzeichnenden Ärztemangels beiträgt - ist absolut ungeeignet, als «Alternative» zur im Parlament diskutierten Aufhebung des Kontrahierungszwangs zu dienen.

Das EDI selbst hat eine Studie veröffentlicht, welche beweist, dass die Bedürfnisklausel keine positiven Auswirkungen hat - deren «Nebenwirkungen» sind uns wohlbekannt!
Weder die eine noch die andere Massnahme, Zulassungsstopp oder Aufhebung der freien Arztwahl, ist geeignet, die Entwicklung der Gesundheitskosten wirksam zu beeinflussen. Denn nicht die Anzahl Leistungserbringer gilt es zu steuern, sondern die effiziente und wirksame Leistungserbringung. Alles andere bleibt Unsinn und läuft Gefahr, sich direkt und unkontrolliert negativ auf die Qualität der Gesundheitsversorgung auszuwirken.

Der VSAO Schweiz bereitet ein Referendum gegen die vorgeschlagene Verlängerung des Zulassungsstopps vor.

Die Unterstützung eines Referendums der FMH gegen die Aufhebung des Kontrahierungszwangs hat der VSAO Schweiz bereits beschlossen.

Wir sind keine Neinsager.

Jasagen oder - noch fataler - Nichtssagen zur Verlängerung des Zulassungsstopps allerdings käme einem «Ja» in Raten zur Aufhebung des Kontrahierungszwangs gleich.

VSAO und FMH werden in diesen Fragen eine gemeinsame Position einnehmen, eine Spaltung der Ärzteschaft bleibt definitiv Wunschdenken weniger Politiker - die Kohärenz der Ärzteschaft ist die Realität. 